\title{
A Case of Atypical Central Skull Base Osteomyelitis with Bilateral Alveolar Bone Destruction
}

\author{
Sohl Park, $\mathrm{MD}^{1}$, Ju Hyun Yun, $\mathrm{MD}^{1}$, Su Jin Kim, $\mathrm{MD}^{1}$, Jung Ho Bae, $\mathrm{MD}, \mathrm{PhD}^{2}$, and So Jeong Kim, MD, $\mathrm{PhD}^{1}$ \\ ${ }^{1}$ Department of Otorhinolaryngology-Head and Neck Surgery, Ewha Womans University, School of Medicine, Mokdong Hospital, Seoul; and \\ ${ }^{2}$ Department of Otorhinolaryngology-Head and Neck Surgery, Ewha Womans University, School of Medicine, Seoul Hospital, \\ Seoul, Korea
}

Central skull base osteomyelitis (CSBO) is a rare, fatal condition seen mostly in elderly diabetic or immunocompromised patients; it mainly involves the sphenoid or occipital bones and diagnosis is often delayed. A 72-year-old male with diabetes was referred to our department with severe pain in the maxillary gingiva. Endoscopic biopsy of the pterygoid bone confirmed the presence of mucormycosis combined with Klebsiella pneumoniae infection. Sinus surgery was performed with bilateral alveolar bone sequestrectomy. Long-term intravenous broad-spectrum antibiotic therapy using meropenem and ampicillin/sulbactam and antifungal therapy using amphotericin B (100 mg/day) was administered; an oral antifungal agent, posaconazole ( $800 \mathrm{mg} / \mathrm{day})$, was also prescribed for 11 weeks. At the 6-month follow-up visit, there were no complications and no progression of the CSBO lesion was observed. Timely diagnosis and multidisciplinary treatment including endoscopic surgery, alveolar bone sequestrectomy, and combined antifungal and antibiotic therapy can reduce mortality and minimize complications associated with CSBO.

KEY WORDS: Skull base $\cdot$ Osteomyelitis $\cdot$ Mucormycosis $\cdot$ Maxilla $\cdot$ Alveolar process.

\section{INTRODUCTION}

Central skull base osteomyelitis (CSBO) is a rare and fatal condition seen mostly in elderly diabetic or immunocompromised patients. Many earlier studies have presented malignant otitis externa, secondary to Pseudomonas aeruginosa infection, as a leading cause of skull base osteomyelitis (SBO) $\left(90-95 \%\right.$ of the cases). ${ }^{12)}$ Atypical SBO arising from the sphenoid or occipital bones without associated external otitis occurs much less frequently and initially may have headache as the only symptom. ${ }^{3)}$ Cranial nerve deficits are a characteristic feature of SBO and is seen in 21$48 \%$ of the patients. ${ }^{2)}$ Owing to the lack of awareness and many differential diagnoses including neoplastic tumours, malignant haemopathies, and granulomatosis, the diagno- sis of CSBO is often difficult. Therefore, timely diagnosis and multidisciplinary treatment is important. Despite the advancements in diagnostic modalities and the introduction of new antibiotics and management techniques, management of SBO continues to be a challenge because of its increasing prevalence and high mortality rate. ${ }^{2)}$

Herein, we report a case of atypical manifestation of CSBO, which was difficult to distinguish from skull base tumours.

\section{CASE REPORT}

A 72-year-old male was referred to our department from the department of oromaxillofacial surgery (OMS), with a four-month history of severe pain in the maxillary gingiva

Received: October 5, 2020 / Revised: December 7, 2020 / Accepted: December 10, 2020

Address for correspondence: So Jeong Kim, MD, PhD, Department of Otorhinolaryngology-Head and Neck Surgery, Ewha Womans University, School of Medicine, 1071 Anyangcheon-ro, Yangcheon-gu, Seoul 07985, Korea

Tel: +82-2-2650-5988, Fax: +82-2-2648-5604, E-mail: entsjkim@gmail.com 
and headache. All maxillary teeth were extracted because of severe swelling of the alveolar bone. He had a history of well-controlled diabetes mellitus. On admission, the patient's vital signs were unremarkable. Neurologic examination revealed no abnormalities. Laboratory tests showed normal white blood cell count $(7,080$ cells $/ \mu \mathrm{L})$, normal erythrocyte sedimentation rate (ESR) $(14 \mathrm{~mm} / \mathrm{h})$, and a normal serum C-reactive protein level $(0.22 \mathrm{mg} / \mathrm{dL})$. Testing for Aspergillus antigen $\beta$-D glucan was negative, and serum IgG, IgA, and IgM levels were also normal. Endoscopic examination revealed necrotic debris with nasal floor destruction in the right nasal cavity (Fig. 1).

Paranasal sinus (PNS) computed tomography revealed extensive destruction of the maxillary alveolar bone with gas densities (Fig. 2A, B). The enhanced PNS magnetic resonance imaging (MRI) revealed an extensive central skull base lesion with bone destruction, and central necrosis with intracranial extension on the right side (Fig. 2C, D).

The department of OMS had previously performed a biopsy of the alveolar bone under local anaesthesia; histopathological examination revealed inflammatory granulation tissue. Therefore, we performed a navigation-guided endoscopic biopsy of the pterygoid bone under general anaesthesia and pathologically confirmed the presence of mucormycosis combined with Klebsiella pneumoniae infection. Alveolar bone sequestrectomy was performed in combination with endoscopic sinus surgery. Necrotic mucosa with large amount of pus discharge were observed on bilateral sphenoid sinus. Destructed inferolateral sphenoid sinus wall was noticed and extended to pterygoid plate.
Navigation guided bone curettage was performed on pterygoid plate. The septa between the sphenoid sinuses on both sides was removed to make it one. Bilateral destructed spongiform alveolar bone was removed transoral approach. After confirming the pathogen, empirically administered intravenous fluconazole $(100 \mathrm{mg} /$ day $)$ was changed to longterm intravenous broad-spectrum antibiotic therapy with meropenem and ampicillin/sulbactam, and antifungal therapy using amphotericin B (100 mg/day). Headache and pain in the maxillary gingiva gradually relieved without complications. At 12 weeks postoperatively, a follow-up MRI showed that osteolysis had subsided and the patient was discharged without any neurologic deficits. An oral antifungal agent, posaconazole ( $800 \mathrm{mg} /$ day), was prescribed for 11 weeks (Fig. 3). At the 6-month follow-up, there were no complications and no progression of the CSBO lesion was observed.

\section{Ethical considerations}

The case report was approved by the Ethics Committee of the Ewha Womans University, Seoul, South Korea. Written informed consent was obtained from the patient.

\section{DISCUSSION}

CSBO mainly involves the sphenoid or occipital bones, without involvement of the temporal bone; its clinical and radiological characteristics are similar to those of $\mathrm{SBO}{ }^{4)}$ Atypical CSBO is an uncommon condition that has significantly high morbidity and mortality. ${ }^{2)}$ The first report of
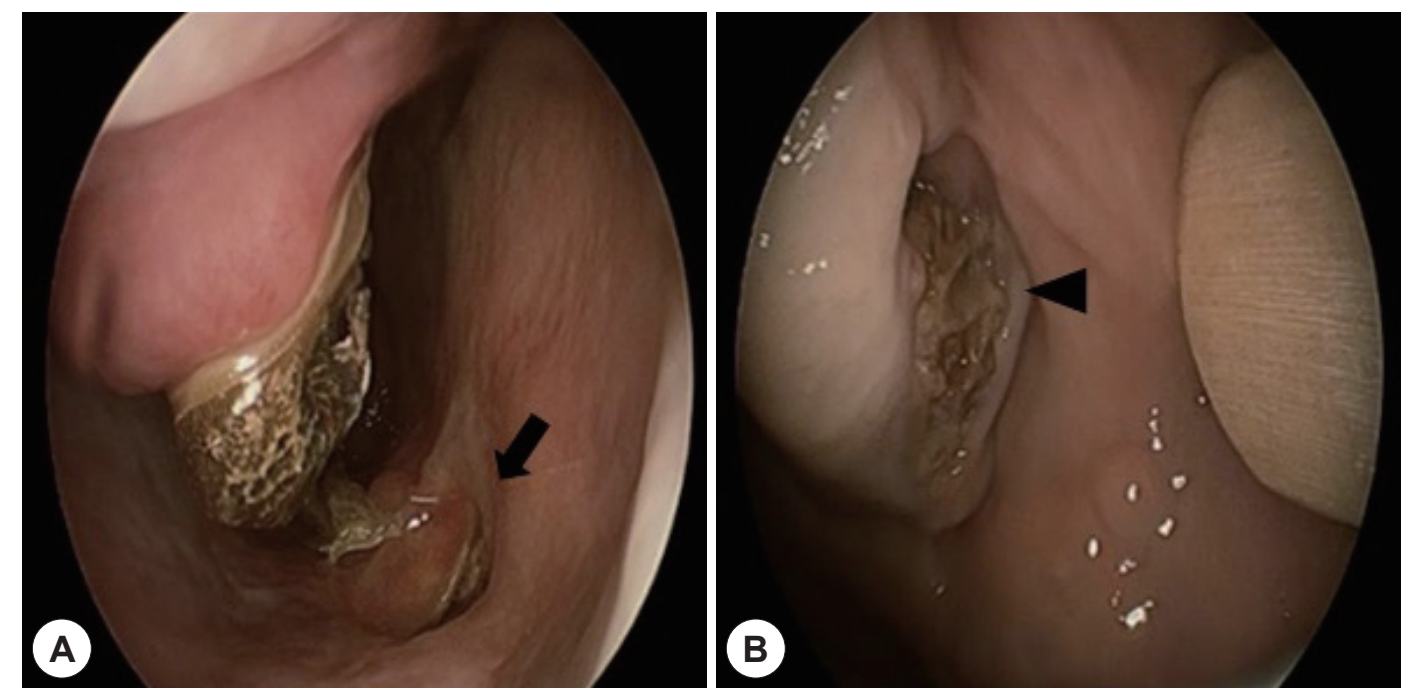

Fig. 1. Endoscopic findings. A: Necrotic debris with nasal floor destruction (arrow) was noted in the right nasal cavity. B: Exposed necrotic alveolar bone with gingival dehiscence (arrowhead). 
SBO in patients without external otitis was made by Sie et al. in $1991^{5)}$ and it is still rare. Shama ${ }^{6}$ and Adams and Bryant $^{7)}$ reported cases of odontogenic origin CSBO, but all patients were unilateral CSBO patients occurred after tooth extraction and dental surgeries. There was no reported case of bilateral CSBO combined with bilateral alveolar bone destruction without cranial nerve paresis.

Partial or incomplete treatment of central or atypical SBO is also responsible for relapse; however, bilateral SBO, as seen in the present case, is extremely rare. ${ }^{4)}$ This case provides details on the diagnosis and management of CSBO with atypical symptoms.

As reported in literature, the most common symptom of CSBO is headache, followed by cranial nerve paresis, nasal discharge, and fever. Cranial nerve paresis indicates progression of the disease. ${ }^{8)}$ In the present case, CSBO had pro-

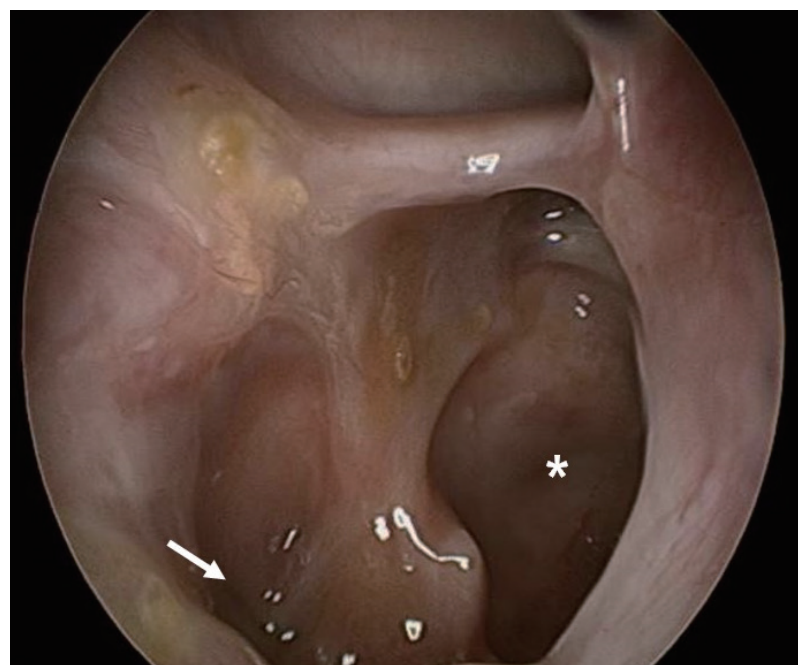

Fig. 3. Endoscopy of the right sphenoid (asterisk) showed mild mucosal swelling without necrotic debris, surgical opening extended to pterygoid plate (arrow).
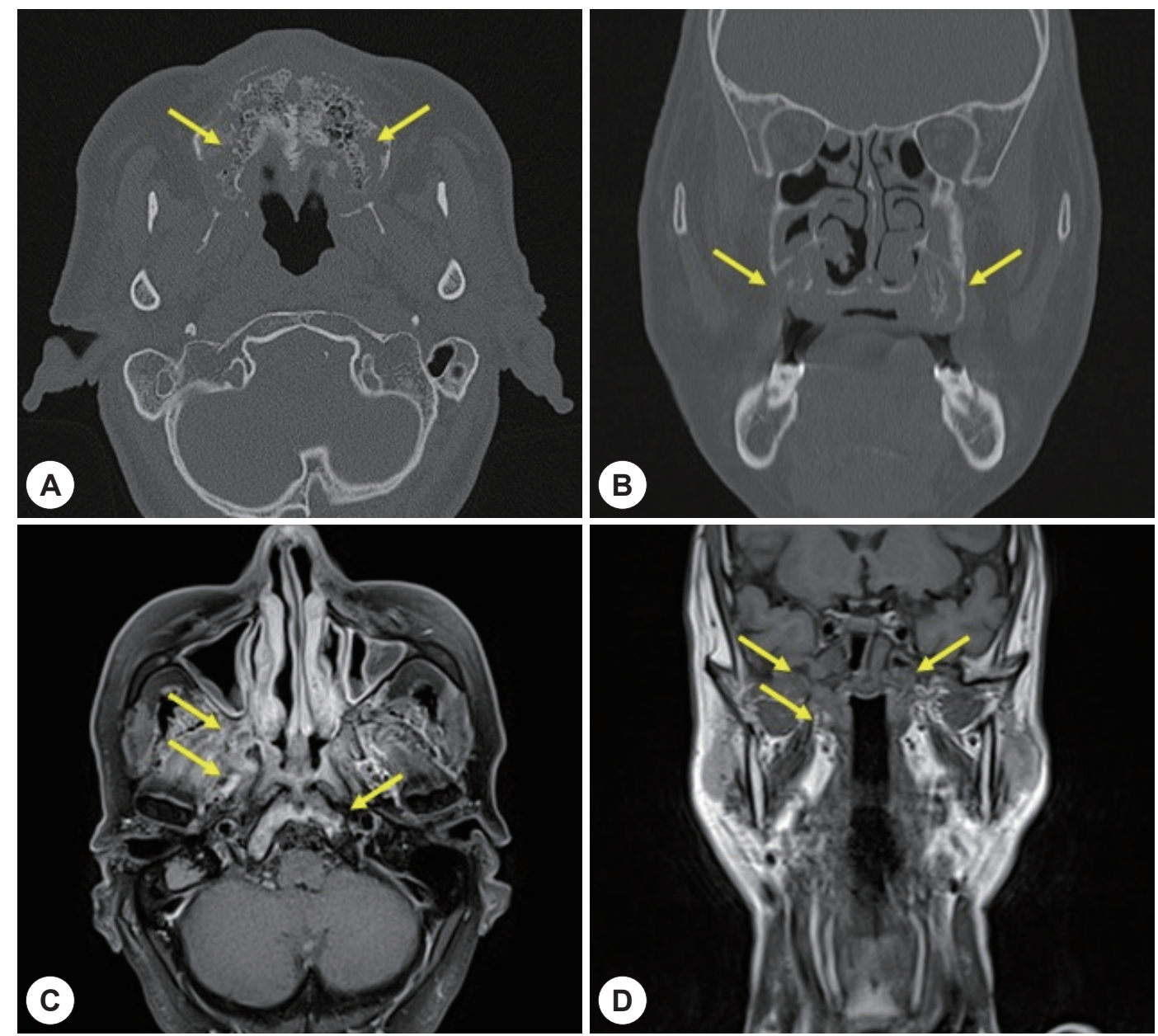

Fig. 2. Preoperative paranasal sinus computed tomography (CT) and magnetic resonance images (MRI). A: Axial CT image shows extensive bone destruction (arrows), with gas densities. B: Coronal CT image shows bilateral osteonecrosis of the alveolar bone (arrows). C: Axial Tl-weighted image shows pathological contrast enhancement in extensive central skull base (clivus, sphenoid body, right greater wing of sphenoid, right pterygoid process of sphenoid, posterior palatine bone) lesion with bone destruction (arrows). D: Coronal T2-weighted image. 
gressed, but cranial nerve paresis was absent, confounding the diagnosis. Occasionally, central or atypical SBO does not manifest with the characteristic signs of infection, i.e., fever, leucocytosis, elevated ESR, and positive blood cultures. ${ }^{9)}$ Invasive fungal infection shows painless, dark-color septal ulcer and crust in endoscopic examination. In this case, the patient also showed ulcerous lesion and dark-colored crust at inferior turbinate, nasal floor and nasal septum. As in the present case, tissue sampling is often required for conclusive diagnosis because imaging studies are nonspecific for central and atypical SBO. In addition, imaging studies can misinterpret SBO as a malignancy. However, considering a diagnosis of CSBO on radiological and clinical basis is significant because waiting for a histopathological diagnosis with biopsy delays treatment. PNS involvement (orbital, oral, or dental) by infection poses a significant risk of cranial osteomyelitis. ${ }^{1011)}$ Therefore, a detailed nasal endoscopic examination is necessary. In this case, although an alveolar bone biopsy was performed earlier, the pathogen was not confirmed; the pathogen was identified after a navigator-guided endoscopic sphenoid and pterygoid biopsy.

CSBO is typically caused by bacteria rather than fungi. Staphylococcus aureus and Pseudomonas aeruginosa are the two most common causative pathogens of CSBO. ${ }^{12)}$ The most common fungus leading to CSBO is Aspergillus. ${ }^{13)}$ However, in this case, the infection was identified as mucormycosis combined with Klebsiella pneumoniae. Because CSBO is rarely caused by fungal infection, empirical antifungal therapy is not recommended. Combination therapy with amphotericin B (100 mg/day) for mucormycosis, and meropenem and ampicillin/sulbactam for Klebsiella pneumoniae infection were administered in this case. In early cases of central or atypical SBO, a 6-20-week course of broad-spectrum antimicrobial agents is recommended. Recurrence of SBO may result in subsequent hospitalisations and a high mortality rate. ${ }^{8}$ Our case suggests that early and aggressive culture-guided, long-term intravenous antifungal agents combined with broad-spectrum antibiotic therapy decreases complications. Aggressive surgical resection of affected tissues will help decrease the infectious and inflammatory load while improving the availability of antimicrobial agents to reach the affected region. ${ }^{2)}$ In this case, early aggressive surgical removal of the infectious sequestra led to good recovery and prevention of neurologic sequelae.

The effective management of this patient with atypical symptoms highlighted the importance of timely diagnosis and appropriate therapy, including surgical resection, antifungal agents, and antibiotics in reducing the mortality and minimising permanent neurologic deficits associated with CSBO.

\section{REFERENCES}

1) Prasad KC, Prasad SC, Mouli N, Agarwal S. Osteomyelitis in the head and neck. Acta Otolaryngol 2007;127(2):194-205.

2) Khan MA, Quadri SAQ, Kazmi AS, Kwatra V, Ramachandran A, Gustin A, et al. A Comprehensive Review of Skull Base Osteomyelitis: Diagnostic and Therapeutic Challenges among Various Presentations. Asian J Neurosurg 2018;13(4):959-70.

3) Singh A, Al Khabori M, Hyder MJ. Skull base osteomyelitis: diagnostic and therapeutic challenges in atypical presentation. Otolaryngol Head Neck Surg 2005;133(1):121-5.

4) Cavel O, Fliss DM, Segev Y, Zik D, Khafif A, Landsberg R. The role of the otorhinolaryngologist in the management of central skull base osteomyelitis. Am J Rhinol 2007;21(3):281-5.

5) Sie KC, Glenn MG, Hillel AH, Cummings CW. Osteomyelitis of the skull base, etiology unknown. Otolaryngol Head Neck Surg 1991; 104(2):252-6.

6) Shama SA. Osteomyelitis of the central skull base: Otogenic and odontogenic sources. Multidetector CT study. The Egyptian Journal of Radiology and Nuclear Medicine 2012;43(4):519-26.

7) Adams JR, Bryant DG. Cranial osteomyelitis: a late complication of a dental infection. British Journal of Oral and Maxillofacial Surgery 2008;46(8):673-4.

8) Sokołowski J, Lachowska M, Karchier E, Bartoszewicz R, Niemczyk K. Skull base osteomyelitis: factors implicating clinical outcome. Acta Neurol Belg 2019;119(3):431-7.

9) Medvedev G, Palacios E, Jones W. Iatrogenic occipital osteomyelitis. Ear Nose Throat J 2009;88(1):720-1.

10) Adams JR, Bryant DG. Cranial osteomyelitis: a late complication of a dental infection. Br J Oral Maxillofac Surg 2008;46(8):673-4.

11) Clayman GL, Adams GL, Paugh DR, Koopmann CF Jr. Intracranial complications of paranasal sinusitis: a combined institutional review. Laryngoscope 1991;101(3):234-9.

12) Johnson AK, Batra PS. Central skull base osteomyelitis: an emerging clinical entity. Laryngoscope 2014;124(5):1083-7.

13) Blyth CC, Gomes L, Sorrell TC, da Cruz M, Sud A, Chen SC. Skullbase osteomyelitis: fungal vs. bacterial infection. Clin Microbiol Infect 2011;17(2):306-11. 